\section{Anomalías dentarias de impacto estético en odontopediatría: Características y tratamiento.}

Abanto $\mathrm{J}^{1}$, Imparato $\mathrm{JCP}^{2}$, Guedes-Pinto $\mathrm{AC}^{3}$, Bönecker $\mathrm{M}^{4}$. Anomalías dentarias de impacto estético en odontopediatría: características y tratamiento. Rev Estomatol Herediana. 2012; 22(3) 171-8.

\section{RESUMEN}

El desarrollo dentario es un proceso que comienza en la vida intrauterina. Los disturbios de desarrollo en este proceso se presentan como anomalías y pueden afectar ambas denticiones, temporal y permanente, teniendo carácter sistémico, local y/o hereditario. Los profesionales que atienden niños deben reconocer y diferenciar características normales de eventuales anomalías de la cavidad bucal que necesitan ser tratadas. Este artículo ofrece a los profesionales del área odontológica una revisión sobre las principales anomalías dentarias directamente relacionadas con la estética del niño y su respectivo tratamiento, con el objetivo de promover una mejor calidad de vida en estos pacientes.

\section{Palabras claves: ANOMALÍAS DENTARIAS, NIÑOS, ESTÉTICA DENTARIA.}

Dental anomalies of esthetic impact in pediatric dentistry: characteristics and treatment

\begin{abstract}
Tooth development is a process that begins in the intrauterine life. The developmental disorders in this process are presented as anomalies and can affect both dentitions, deciduous and permanent, having a systemic, local and/or hereditary character. Professionals who treat children must recognize and differentiate normal characteristics of possible abnormalities in the oral cavity that need to be treated. This article provides to dental professionals a review of the major dental abnormalities directly related to the aesthetic of children and their respective treatment, with the aim to promote a better quality of life in these patients.
\end{abstract}

Key words:: ABNORMALITIES, CHILDREN, DENTAL ESTHETIC.

\author{
Jenny Abanto ${ }^{1}$ \\ José C. Pettorossi Imparato ${ }^{2}$ \\ Antônio C. Guedes-Pinto ${ }^{3}$ \\ Marcelo Bönecker ${ }^{4}$
}

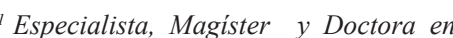
Odontopediatría de la Facultad de Odontología de la Universidad de São Paulo - USP. Profesora del Curso de Odontopediatría en la Primera Infancia de la FUNDECTO-USP. Sao Paulo, Brasil.

2 Profesor del Departamento de Ortodoncia y Odontopediatría de la Facultad de Odontología de la Universidad de São Paulo - USP. Sao Paulo, Brasil.

${ }^{3}$ Ex-Profesor Titular del Departamento de Ortodoncia y Odontopediatría de la Facultad de Odontología de la Universidad de São Paulo - USP. Sao Paulo, Brasil.

${ }^{4}$ Profesor Titular del Departamento de Ortodoncia y Odontopediatría de la Facultad de Odontología de la Universidad de São Paulo - USP. Sao Paulo, Brasil.

\section{Correspondencia:}

Profa. Dra. Jenny Abanto.
Disciplina de Odontopediatria. Faculdad de
de Odontologia.
Universidade de São Paulo. Avenida
Professor Lineu Prestes, 2227 Cidade
Universitária. São Paulo-SP, Brasil - CEP:
05508000.
Telf.: 55 (11) $3091-7835$ Fax: 55 (11) 3091-
7854 / E-mail: jennyaa@usp.br

\section{Introducción.}

La sonrisa desempeña una función importante en la vida de adultos, adolescentes y niños, siendo importante para su bienestar general y emocional. Asimismo, una sonrisa saludable está relacionada con el atractivo físico, que representa un papel importante en la forma como nos vemos, sentimos y de cómo somos vistos por los otros (1). De acuerdo con la psicología infantil, una sonrisa saludable es un medio por el cual los niños comienzan a formar relaciones interpersonales y la autoestima (2). Sin embargo, ni siempre todos los niños pueden sonreír como les gustaría, eso debido a la presencia de anomalías dentarias que los colocan en desventaja psicosocial; perjudicando su estética, sonrisa y afectando su calidad de vida (3). Este hecho ha generado un aumento significativo del tratamiento estético y de una sonrisa saludable en la consulta odontopediátrica en los últimos años.

El cirujano dentista que es clínico general, tanto como el odontepediatra atienden, con relativa frecuencia, pacientes portadores de diversas anomalías dentarias, estando en sus manos la responsabilidad de realizar el diagnóstico y tratamiento de la anomalía y/o derivarla (4).

Este artículo de revisión tiene como objetivo describir las principales anomalías dentarias, originadas en el período de odontogénesis, que pueden interferir con la estética de los niños. Los autores tratan de enfatizar el diagnóstico, etiología y opciones de tratamientos para cada caso con la finalidad de promover una mejor calidad de vida en estos pacientes.

\section{Odontogénesis:}

El conocimiento de la odontogénesis es fundamental para la comprensión de los disturbios de crecimiento y desarrollo dentarios. Por este motivo, serán descritos brevemente los principales eventos que ocurren en tal proceso.

El proceso de desarrollo dentario, denominado odontogénesis, es un proceso fisiológico de evolución continua, en el cual los cambios histológicos, fisiológicos y bioquímicos tienen lugar simultáneamente y de forma progresiva (5). La formación y la diferenciación del 
órgano dentario son realizadas en varios periodos de desarrollo que siguen la siguiente secuencia (5):

Crecimiento: iniciación; proliferación; histodiferenciación; morfodiferenciación y aposición.

\section{Mineralización.}

Erupción: Estos periodos son sensibles a la inducción de factores modificadores que pueden ocasionar anomalías clínicamente diferentes. El cuadro 1 muestra diversas anomalías que pueden producirse durante cada periodo del desarrollo dentario. De éstas, sólo las principales anomalías que tienen un impacto estético serán abordadas en este artículo.

Anodoncia: El término anodoncia dentaria es empleado genéricamente para indicar la ausencia congénita de uno o más dientes.

La anodoncia pude ser clasificada en parcial o total $(5,6)$. La ano- doncia parcial (hipodoncia u oligodoncia) envuelve uno o más dientes y es una condición bastante común, principalmente en la dentición permanente.

La anodoncia total, en la cual todos los gérmenes dentarios están ausentes, ocurre tanto en la dentición temporal como la permanente, no obstante, es una condición rara y cuando ocurre, puede estar asociada a un disturbio más generalizado. La anodoncia es más común en la dentición permanente, ocurriendo en aproximadamente $2-10 \%$ en diferentes poblaciones (excluyendo la ausencia de terceros molares), comparada con la dentición temporal, en la que la prevalencia es menor que 1\% (6). Esta anomalía es predominante en el sexo femenino, siendo que los dientes incisivos laterales superiores (Figuras 1A y 1B) e incisivos inferiores son los más frecuentemente afectados $(5,7)$.

\begin{tabular}{|c|l|}
\hline Iniciación y Profiferación Celular & $\begin{array}{l}\text { Estado de Brote y Casquete } \\
\text { Agenesia, supernumerarios, odontomas, geminación y fusión. }\end{array}$ \\
\hline $\begin{array}{c}\text { Histodiferenciación } \\
\text { Celular }\end{array}$ & $\begin{array}{l}\text { Estadio de Campana. } \\
\text { Amelogenesis imperfecta tipo hipoplásica, dentinogenesis } \\
\text { imperfecta. }\end{array}$ \\
\hline $\begin{array}{c}\text { Morfodiferenciación } \\
\text { Celular }\end{array}$ & $\begin{array}{l}\text { Estadio de Campana. } \\
\text { Microdoncia, macrodoncia, dens in dens, taurodentismo, raices } \\
\text { supernumerarios. }\end{array}$ \\
\hline Aposición & Hipoplasia del esmalte, perla del esmalte \\
\hline Mineralización & Amelogenesis imperfecta tipo hipomineralizada. \\
\hline Erupción & Aanquilosis, impactación, atraso y erupción prematura. \\
\hline
\end{tabular}

Cuadro 1. Anomalias que ocurren durante la odontogénesis.

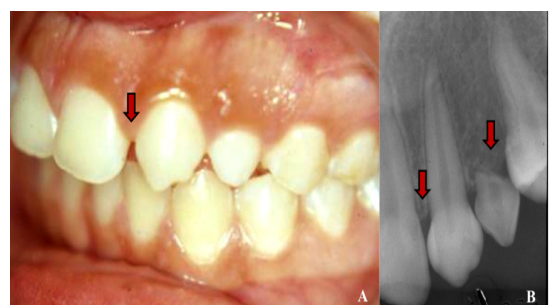

Fig 1A y 1B. A. Anodoncia del diente 22.B. Retención prolongada del diente 63 conreabsorción total de la raiz.
Wong et al. (8), mostraron que la hipodoncia en niños está moderadamente asociada con una peor calidad de vida y baja autoestima en escolares, sugieren que mantener los dientes temporales predecesores en la arcada dentaria sería una opción viable de tratamiento en estos pacientes, al decidir esta opción de tratamiento se debe considerar que el diente temporal mantenido en boca debe estar sin signos de anquilosis y sin reabsorción de sus raíces (9). Las figuras 1A y 1B muestran el caso de un paciente con anodoncia del diente 22 y retención prolongada del diente 63 , a pesar de que el canino temporal está presente, éste no puede ser mantenido en boca ya que muestra reabsorción de sus raíces. La extracción del canino temporal y el tratamiento ortodóncico, para reposicionar el canino permanente, y protésico, deberán ser realizados para cerrar el espacio y recuperar la función y estética del segmento anterior, mantener dientes temporales sin movilidad (9), ó prótesis fija con retenedor que no interfiere en el crecimiento de los maxilares; tratamiento ortodóncico y en un futuro implantes osteointegrados $(6,10)$.

Supernumerarios: Son dientes adicionales a aquellos de la serie normal. Éstos pueden originarse de la división del germen dentario o germen de la lámina dentaria $(5,11)$.

La mayoría de los casos presenta etiología desconocida.

El diente supernumerario puede ser dividido de acuerdo con su forma en dos tipos: si el diente supernumerario se asemeja morfológicamente a un diente normal, éste se denomina "suplementario", si presenta una forma y tamaño anormal es denominado "rudimentario" $(11,12)$.Ocurren frecuentemente en las regiones anteriores.

Cuando tienen la forma conoide y está localizado en la línea mediana, entre los incisivos superiores, es denominado mesiodens o mesio-

diente (13) (Figura 2). 


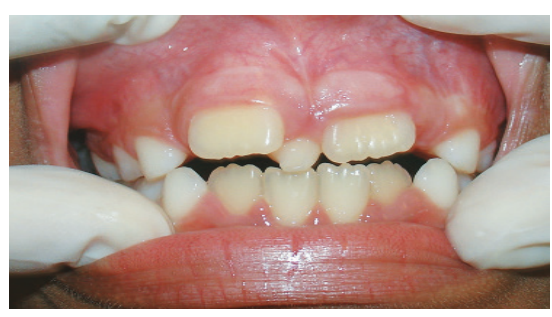

Fig 2. Mesiodens en la dentición permanente con forma conoide afectando la estética.

Raras veces son encontrados en la mandíbula. Los dientes supernumerarios generalmente son únicos y ocurren en aproximadamente $0,8-3,8 \%$ de la población en la dentición permanente. Son menos frecuentes en la dentición temporal (12)(Figuras 3A y 3B). Ocasionalmente están asociados a otros defectos, como fisura labio-palatina, displasia cleidocraneal, Síndrome de Gardner y Síndrome oro-facialdigital $(5,11,13)$ (Figura 2).

Cuando alguna de las complicaciones descritas anteriormente ocurre, está indicada la remoción quirúrgica del supernumerario, tan pronto como sea posible. No es recomendable extraer el supernumerario cuando no está causando ninguna interferencia en la erupción y oclusión de los otros dientes, atraso o reabsorción de dientes vecinos o no está provocando dolor por la compresión $(6,11)$. En esos casos es mejor esperar la erupción y después remover quirúrgicamente (13).

Odontomas: Son tumores odontogénicos mixtos, derivados de los tejidos que forman los dientes, el término odontoma es usado para designar una anomalía de desarro11 no neoplásica que contiene esmalte y dentina (5). La etiología es desconocida, estos tumores pueden ser clasificados de dos formas $(5,6)$ :

Odontoma compuesto: masa formada por varias estructuras pequeñas y discretas semejantes a dientes, denominadas dentículos.
Cada dentículo consiste en un esmalte, dentina, cemento y pulpa dispuestos de manera normal. El odontoma compuesto se presenta predominantemente en la región anterior del maxilar. Radiográficamente puede aparecer como una lesión con imagen mixta (radiopaca y radiolúcida) con distinto dentículo identificable.

Odontoma complejo: masa formada por tejidos dentarios desordenadamente distribuidos, ocurre frecuentemente en la región de molares en la mandíbula.

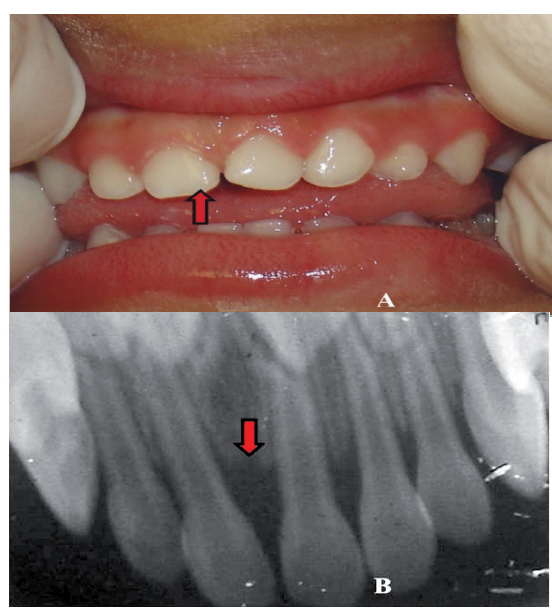

Fig 3A y 3B A. Incisivo central superior supernumerario en la dentición temporal. B. Radiográficamente es dificil distinguir el diente supernumerario.

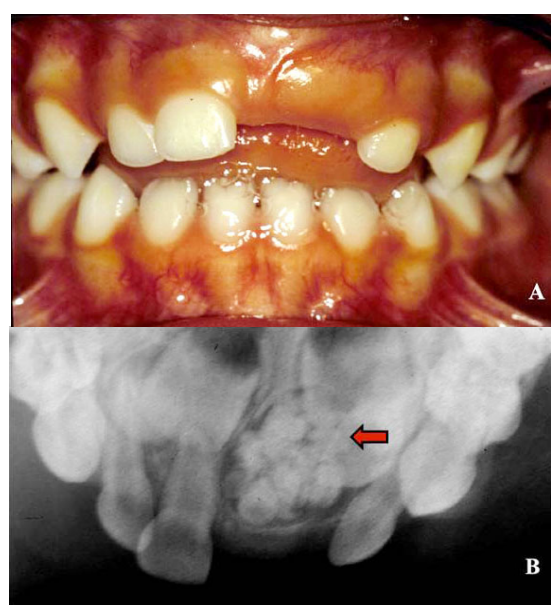

Fig 4A y 4B. A: Abultamiento óseo e impactación del diente 61 ocasionando. B: Radiográficamente se observa un odontoma compuesto.

El principal problema estético de esta anomalía es ocasionado por el odontoma compuesto debido a su localización en la región anterior de la arcada. Los odontomas no permiten la erupción del diente temporal o del permanente sucesor ocasionando la ausencia del diente y consecuentemente un espacio libre o diastema amplio en la arcada (Figuras 4A y 4B). El tratamiento del odontoma es su remoción quirúrgica total, para evitar cualquier posibilidad de recidiva (6).

Fusión: Es la unión entre la dentina y/o esmalte de dos o más dientes desarrollados separadamente. Esta anomalía es observada con frecuencia en la región anterior y en la dentición temporal (15). Radiográficamente, se obsrva dos dientes con dos coronas, dos raíces y dos canales radiculares. La fusión puede ser de un elemento de la serie normal y otro supernumerario, pero puede ocurrir también entre dos dientes de la serie normal. La fusión de dientes temporales envolviendo dos dientes adyacentes, principalmente el incisivo central y lateral superior o el incisivo lateral y canino inferior son mucho más frecuentes que cualquier otro diente fusionado $(6,7)$.

El tratamiento de los dientes fusionados puede ser complejo y diversos protocolos de tratamiento pueden ser realizados, incluyendo, tratamiento endodóncico, quirúrgico e intervenciones periodontales. Algunos estudios optan por la extracción del diente anómalo, seguido de un tratamiento ortodóncico para alcanzar una estética y oclusión ideal. Hay dos situaciones principales donde esto se recomienda $(15,16)$ :

Cuando hay fusión de un diente permanente con un supernumerario, siendo que los dientes presentan raíces completamente separadas. En los casos de problemas estéticos, 
funcionales y ortodóncicos, la separación quirúrgica (hemisección intrabucal) es recomendada si los dientes muestran raíces separadas. La hemisección extraoral y la reimplantación inmediata (hasta 5 minutos) puede ser también una alternativa en los casos de fusión de un diente permanente con un supernumerario.

Cuando 2 dientes permanentes se han fusionado, hay una única cámara pulpar y conductos radiculares independientes. En esta situación, el tratamiento endodóncico de ambos canales es indicado, con una apertura doble de la cámara pulpar, sellado del conducto radicular y seguido por la división de las coronas para separar los dientes y restauraciones coronarias con resinas compuestas o coronas estéticas.

Los dientes fusionados presentan también surcos en sus divisiones, así la limpieza se hace difícil pudiendo ocasionar lesiones de caries que afectan la estética (Figura $5 \mathrm{~A}$ y $5 \mathrm{~B})$. Un hallazgo frecuente en la fusión de dientes temporales es la ausencia congénita de uno de los dientes permanentes correspondientes (16), el cual puede causar problemas de estética debido al espacio generado en la arcada.

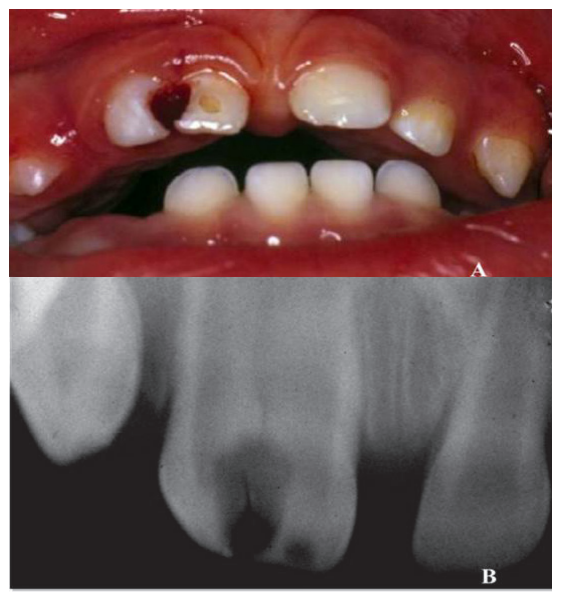

Fig 5A y 5B. A: Fusión del diente 51 y 52 ocasionando lesión de caries en su unión. B: La radiografia muestra la unión entre dos dientes desarrollados separadamente caracterizando la fusión.
Geminación :Es la división de un germen dentario en dos. Radiográficamente las geminaciones se presentan con una raíz y un canal radicular, pero, con dos coronas y dos cámaras pulpares $(5,15)$ (Figuras 6 A y 6B). Muchas veces no es posible realizar el diagnóstico radiográfico diferencial entre la fusión y la geminación.

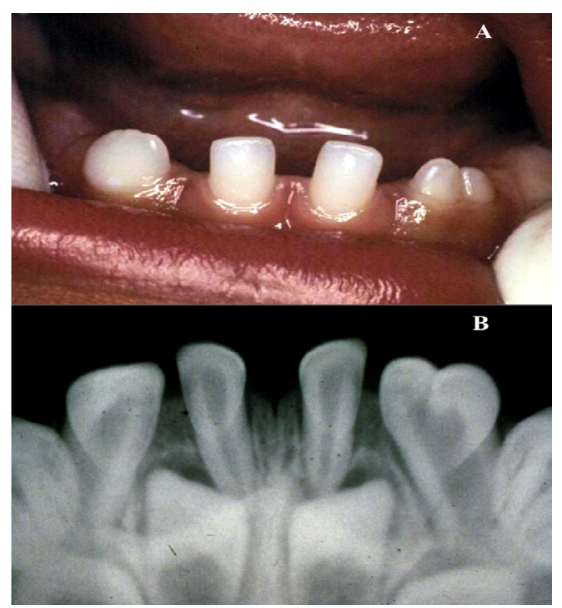

Figura 6A y 6B. A: Apariencia clínica de la geminación del diente 72. B: Radiográficamente se observa una raiz y un canal radicular con dos coronas y dos cámaras pulpares.

La ocurrencia de la fusión y geminación puede ser unilateral o bilateral (15). Ambas son más comunes en la dentición temporal que permanente y ocurren principalmente en la región anterior $(6,16)$. El impacto estético de la geminación es muy similar al de la fusión por las características clínicas similares que ambas poseen. El tratamiento de la geminación consiste básicamente en la reducción del diámetro mesiodistal de la corona cuando ésta es muy ancha, con el objetivo de promover el normal desarrollo de la oclusión (6). Cuando la corona no es ancha, el desgaste periódico con disco abrasivo es más indicado. El tratamiento para los surcos divisorios con o sin lesión de caries es similar al de la fusión.
Amelogénesis imperfecta tipo hipoplásica: Constituye un grupo de condiciones hereditarias que afectan la formación del esmalte. La amelogénesis imperfecta tipo hipoplásica está relacionada con la producción inadecuada de la matriz del esmalte $(5,17)$. Todos los tipos de amelogénesis imperfecta afectan ambas denticiones: temporal y permanente, la mayor parte del esmalte está afectado en todos los dientes, siendo que el esmalte no alcanza la espesura normal y ocurre una variación considerable en los aspectos clínicos $(5,17)$. Esta anomalía produce un gran impacto negativo en la estética debido a sus características clínicas. En algunos casos, el esmalte puede presentar rugosidad y fosas o surcos verticales e irregulares $(5,6)$ (Figura 7).

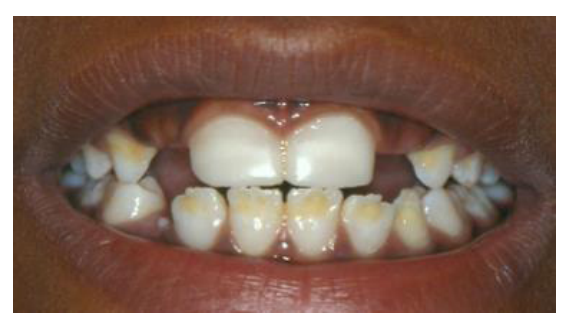

Figura 7. Amelogénesis imperfecta tipo hipoplásica. Clínicamente se observa el esmalte amarillento con fosillas irregulares, cúspides afiladas y consistencia dura. Los dientes 51 y 61 recibieron coronas de acetato con resina compuesta para recuperar la estética.

En su forma lisa, el esmalte sobre toda la corona es afectado y los dientes presentan cúspides afiladas. Clínicamente, los dientes afectados presentan coronas de color ámbar, lisas, brillantes, duras y ausencia de contactos interproximales $(5,16,17)$. Con los avances en las técnicas y materiales adhesivos, es posible realizar restauraciones directas con resinas compuestas para restablecer la función y estética, inclusive en los casos con exposición extensa de la dentina (18). El tratamiento puede tener hasta tres fases distintas: el tratamiento de 
emergencia provisional en los dientes temporales y permanentes para aliviar la sintomatología dolorosa; el tratamiento transitorio en la fase de dentición mixta $y$, por fin, el tratamiento rehabilitador final en los dientes permanentes en la fase adulta (19). En la dentición temporal el uso de coronas de celuloide con resinas compuestas (Figura 7) o restauraciones directas de resina $\mathrm{y} / \mathrm{o}$ cementos ionómeros de vidrio modificados por resina son las más usadas. En el caso de dientes permanentes, el uso de coronas metalcerámicas o de resinas compuestas indirectas para los dientes posteriores y anteriores son muy usados, sin embargo, este tipo de tratamiento exige preparaciones dentarias para cumplir los requisitos de resistencia y retención $(6,17,19)$.

Dentinogénesis imperfecta: Comúnmente denominada como dentina opalescente hereditaria. Son conocidos tres tipos de dentinogénesis imperfecta $(5,20)$ : Tipo I, que está asociada con la osteogénesis imperfecta; Tipo II, donde apenas los dientes son afectados; e Tipo III, que ocurre apenas en un grupo raro y aislado en los EEUU. El tipo II es el más común, aunque esta anomalía es relativamente rara.

Afecta estéticamente tanto a la dentición temporal como a la permanente, clínicamente, los dientes presentan un color que varía del castaño amarillento al gris azulado, pero todos presentan un aspecto opalescente o translúcido (5). Radiográficamente presenta raíces cortas y delgadas, con obliteración parcial o total de las cámaras pulpares y canales radiculares $(6,20)$.

Puede haber pérdida precoz del esmalte y consecuentemente una atrición marcada por no tener so- porte dentinario.

El tratamiento de esta anomalía es complejo para ambas denticiones. La colocación de coronas de acero en los dientes temporales posteriores para restablecimiento de la dimensión vertical (20) y de coronas de acetato con resinas compuestas en los dientes anteriores es la más indicada para prevenir la atrición marcada de los dientes. La utilización de overdentures es indicada en casos de remanentes que están en buenas condiciones clínicas para ser mantenidos en la cavidad bucal (21). En casos más graves de dentinogénesis imperfecta en la dentición temporal, donde hay atrición total de los dientes, la colocación de mantenedores estético-funcionales removibles sobre remanentes temporales en buenas condiciones, contribuiría a mantener la guía de erupción de los sucesores permanentes. Este procedimiento es recomendado con un tratamiento endodóncico previo de los dientes remanentes.

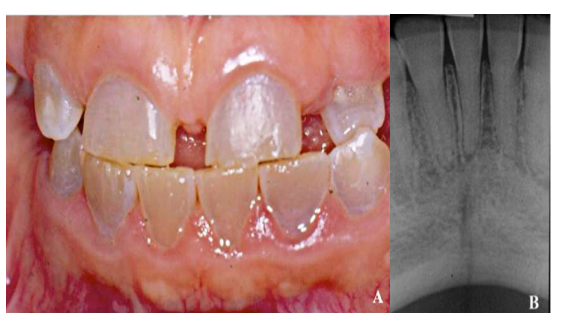

Fig 8A y 8B. A: Dentinogénesis imperfecta en la dentición permanente. Clínicamente, se observan los dientes de color castaño amarillento y aspecto opalescente o translúcido. B: Radiográficamente se observa obliteración total das cámaras pulpares y canales radiculares.

Microdoncia: Los dientes son más pequeños que el normal debido al insuficiente desarrollo del germen dentario. Existen tres tipos de microdoncia $(5,22,23)$ : microdoncia generalizada verdadera, en la que todos los dientes son más pequeños que el normal, esta condiciónes extremamente rara; microdoncia generalizada relativa, en la que los dientes son normales o levemente más pequeños, se encuentran en maxilares más grandes dando una apariencia de microdoncia; y microdoncia localizada, que envuelve un único diente. La microdoncia localizada es más rara en la dentición temporal que en la permanente (6) (Figura 9).

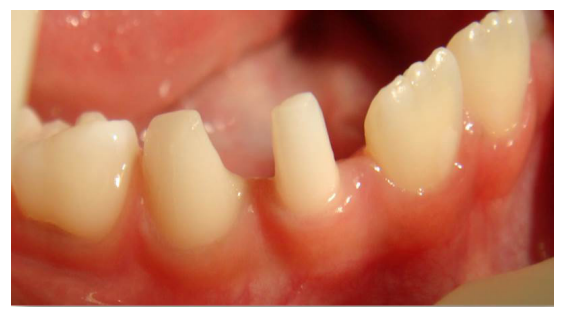

Figura 9. Microdoncia del diente 82.

Macrodoncia: Es uno o varios dientes grandes que el normal, los factores etiológicos son atribuidos a la herencia y a causas idiopáticas. La macrodoncia puede ser clasificada en $(5,6,23)$ : macrodoncia verdadera generalizada, cuando todos o por lo menos la mayoría de los dientes son mayores que lo normal; macrodoncia generalizada relativa, que es el resultado de la presencia de dientes normales o ligeramente más grandes que lo normal en maxilares pequeños; y macrodoncia localizada, cuando solamente un diente es mayor que lo normal.

El tratamiento es simple y consiste en la disminución del ancho mesiodistal del macrodiente y en el aumento del ancho mesiodistal de su homólogo con resina compuesta o uso de carillas (6). Si hubiera diastema, se puede optar por su cierre por medio del tratamiento ortodóncico para promover una estética favorable para el paciente.

Hipoplasia del esmalte: La hipoplasia del esmalte es definida como la formación incompleta o defectuosa de la matriz orgánica del esmalte dental (5). Afectar tanto 
la dentición temporal como la permanente, la hipoplasia envolviendo un único diente es más común en incisivos superiores permanentes, $o$ en premolares superiores e inferiores. La causa más frecuente de esta anomalía es la infección o trauma asociado a los dientes temporales predecesores, produciendo un daño en los ameloblastos de los permanentes sucesores $(5,6)$. En estos casos, los dientes son generalmente denominados Dientes de Turner. En algunos casos la anomalía puede estar asociada a otras condiciones, tales como (24), trauma al nacimiento debido al uso de intubación traqueal; complicaciones durante y después del parto; infección o traumatismos locales; irradiación; terapia antineoplásica; hiperbilirrubinemia; deficiencias de vitaminas $\mathrm{A}, \mathrm{C}$ e $\mathrm{D}$, además de calcio y fósforo; enfermedades metabólicas que incluyen enfermedades cardiacas congénitas, renales, desórdenes neurológicos e hipocalcemia. La sífilis congénita también produce alteraciones hipoplásicas características en el esmalte de los incisivos y molares permanentes debido a la infección del germen dentario por espiroquetas (5). Los incisivos superiores presentan los bordes en escotadura o semilunar oblicuos hacia la línea media, a veces ensanchados en su base, junto a la encía, por lo que se les denomina "dientes de destornillador" o "dientes en tonel". Las superficies y los tercios oclusales de las coronas de los primeros molares son cubiertas por pequeñas masas globulares de esmalte ("molares en mora"). Clínicamente, las alteraciones hipoplásicas varían de una pigmentación amarillenta o castaña del esmalte (Figuras 10A y 10B) hasta excavaciones extensas e irregular de la superficie.

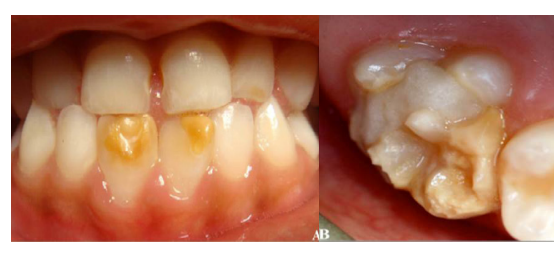

Fig 10. A: Hipoplasia de los dientes 31 y 41 debido a traumatismo en los predecesores temporales. B: Hipoplasia del diente 16.

Es muy importante hacer el diagnóstico diferencial entre las hipoplasias y las hipomineralizaciones. Estas últimas son defectos cualitativos de los tejidos dentarios, mientras que las hipoplasias son defectos cuantitativos envolviendo la superficie del esmalte (5). El tratamiento es similar en ambas.

Con relación al tratamiento, éste debe ser lo menos invasivo posible. La mayoría de estos pacientes presentan problemas de comportamiento relacionados a múltiples consultas de tratamiento, ya que muchos de ellos fueron inadecuadamente anestesiados o fueron tratados sin anestesia local, principalmente en los casos de hipoplasias en molares permanentes (25). La evaluación del riesgo de caries, el diagnóstico precoz de la anomalía, la remineralización y desensibilización con flúor tópico, la prevención de lesiones de caries y rupturas post-eruptivas del esmalte, el tratamiento restaurador o extracción, y el mantenimiento, son los 6 pasos de manejo propuestos para las hipoplasias (25).

Como la adhesión de los ionómeros es baja, éstos deben ser reemplazados por sellantes resinosos tan pronto el diente erupcione totalmente. En casos de restauraciones operatorias, tanto la remoción de todo el esmalte defectuoso hasta que una superficie sana sea encontrada, como sólo la remoción del esmalte poroso hasta encontrar una resistencia en el diente con la son- da, son opciones descritas por una revisión sistemática (25). El uso de cementos ionómeros de vidrio, como material intermedio previo a la colocación de la resina compuesta, también ha sido propuesto en casos donde la cavidad envuelve áreas extensas de dentina (26).

La microabrasión del esmalte en dientes anteriores representa otra opción de tratamiento en el caso que no haya sensibilidad y en lesiones menos profundas. El algunos casos, se puede realizar la microabrasión antes de la colocación del material resinoso en estos dientes. En casos más graves de hipoplasia, la extracción puede ser considerada, teniendo en cuenta la edad del paciente, consideraciones ortodóncicas, presencia de otras anomalías, compromiso pulpar, durabilidad de las restauraciones y costo esperado del tratamiento a largo plazo $(25,26)$.

Fluorosis dentaria : El desarrollo de la fluorosis depende de la cantidad total de flúor ingerida de diversas fuentes, de su duración y tiempo de exposición. Clínicamente, se caracteriza por un ligero moteado blanquecino del esmalte, manchas o estrías blancas, o en los casos más graves, por una decoloración amarillenta o negra-castaña, particularmente en los dientes más expuestos a la luz $(5,27)$.

El tratamiento de la fluorosis dentaria es básicamente estético debido a sus características clínicas y pueden variar de un blanqueamiento y/o microabrasión del esmalte en los casos más leves; restauraciones con resina compuesta o ionómeros de vidrio modificados por resina en los casos de fluorosis moderada y el uso de coronas de porcelana en los casos más graves $(27,28)$. 
Amelogénesis imperfecta tipo hipomineralizada: Es una anomalía hereditaria común de este tipo de alteraciones, está relacionada a la mineralización inadecuada del esmalte dentario. Los dientes recién erupcionados parecen normales en tamaño y forma y tienen esmalte de espesor normal. Sin embargo, el esmalte tiene una consistencia de tiza blanda y presenta un aspecto que varía de blanco opaco hasta una apariencia mosqueada de color amarillo-castaño $(5,6)$, afectando la estética (Figura 11). Debido a su mineralización deficiente, el esmalte presenta una densidad similar a la de la dentina en las radiografías $(5,29)$. El esmalte presenta consistencia blanda y se pierde poco después de la erupción dentaria, dejando la dentina expuesta y altamente sensible (6).

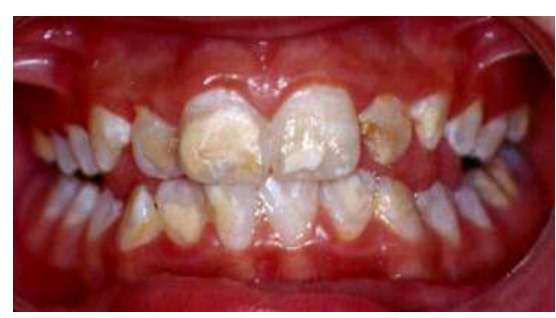

Fig 11. Amelogénesis imperfecta tipo hipomineralizada en la dentición permanente. Clínicamente, se observa el esmalte con consistencia blanda y color blanco opaco con apariencia mosqueada amarillenta.

Consideraciones finales: Teniendo en cuenta el impacto estético que algunas anomalías de desarrollo dentario pueden causar a los niños, se recomienda que el cirujano dentista u odontopediatra conozcan en detalle la etiología y diagnóstico descritos en este artículo, con el objetivo de escoger el tratamiento odontológico más adecuado para cada alteración y paciente.

\section{Referencias Bibliográficas.}

1. Jenny J, Cons NC, Kohout FJ, Jacobsen JR. Relationship between dental aesthetics and attributions of self-confidence. J Dent Research. 1990; 69:204.

2. Rebok G, Riley A, Forrest C, Starfield B, Green B, Robertson J, Tambor E. Elementary school-aged children's reports of their health: a cognitive interviewing study. Qual Life Res .2001; 10(1): 59-70.

3. Barbosa TS, Gaivão MB. Oral health-related quality of life in children: part II. Effects of clinical oral health status. A systematic review. Int J Dent Hyg. 2008;6(2):100-7.

4. Abanto JA, Raggio DP, Alves FBT, Corrêa FNP, Corrêa MSNP, Bönecker M. Characteristics of newborns: report of some oral anomalies and their treatment. IJD. 2009; 8(3): 140-45.

5. Soames JV, Southam JC. Patologia oral. 4a ed. Rio de Janeiro: Guanabara Koogan, 2008.p. 3-13.

6. Campos V, Cruz RA, Mello HS. Diagnóstico e tratamento das anomalias da odontogênese. $1^{\mathrm{a}}$ ed. São Paulo: Livraria Santos, 2004. p. 3-77.

7. King NM, Tongkoom S, Itthagarun $\mathrm{A}$, Wong HM, Lee CK. A catalogue of anomalies and traits of the primary dentition of southern Chinese. J Clin Pediatr Dent. 2007; 32(2):139-46.

8. Wong AT, McMillan AS, McGrath C. Oral health-related quality of life and severe hypodontia. J Oral Rehabil. 2006; 33(12): 869-73.

9. Sabri R. Management of overretained mandibular deciduous second molars with and without permanent successors. World J Orthod. 2008; 9(3):209-20.

10.Marianetti TM, Azzuni C, Moro A, Cervelli D, Gasparini G, Boniello R, Pelo S.Case report of oligodontia: long term stability of orthognatic surgery and prosthetic rehabilitation. Minerva Stomatol. 2011;60(3):139-47.
11.Parolia A, Kundabala M, Dahal M, Mohan M, Thomas MS. Management of supernumerary teeth.J Conserv Dent. 2011;14(3):221-4.

12.Leco MI, Martín JF, Martínez JM. An observational study of the frequency of supernumerary teeth in a population of 2000 patients. Med Oral Patol Oral Cir Bucal. 2007;12(2):134-8.

13.Cogulu D, Yetkiner E, Akay C, Seckin O, Alpoz R. Multidisciplinary management and longterm follow-up of mesiodens: a case report. J Clin Pediatr Dent. 2008; 33(1):63-6.

14.Baldawa RS, Khante KC, Kalburge JV, Kasat VO. Orthodontic management of an impacted maxillary incisor due to odontoma. Contemp Clin Dent. 2011;2(1):37-40.

15.Tuna EB, Yildirim M, Seymen F, Gencay K, Ozgen M. Fused teeth: a review of the treatment options. J Dent Child (Chic). 2009;76(2):109-16.

16. Karacay S, Guven G, Koymen R. Management of a fused central incisor in association with a macrodont lateral incisor: A case report. Pediatr Dent. 2006;28(4):336-40.

17.Ayers KM, Drummond BK, Harding WJ, Salis SG, Liston PN. Amelogenesis imperfecta: multidisciplinary management from eruption to adulthood. Review and case report. N Z Dent J. 2004;100(4):101-4.

18.Gelmalmaz D, Isik F, Keles A, Kükrer D. Use of adhesively inserted full-ceramic restorations in the conservative treatment of amelogenesis imperfecta: a case report. J Adhes Dent. 2003; 5(3):235-242. 
19.Ozturk N, Sari Z, Ozturk B. An interdisciplinary approach for restoring function and esthetics in a patient with amelogenesis imperfecta and malocclusion: A clinical report. J Prosthet Dent 2004; 92(2):112-4.

20.Freitas KP, Antonio AG, Winz MLP, Castro RAL, Vianna RBC. Dentinogênese imperfeita tipo III e tipo II em crianças: Relato de Casos. Rev Odonto 2008; 16:130-6.

21.Goud A, Deshpande S. Prosthodontic rehabilitation of dentinogenesis imperfecta. Contemp Clin Dent. 2011;2(2):138-41.

22.Mehrotra KK, Saimbi CS, Jha T. Microdontic maxillary lateral incisor: case report. J Clin Pediatr Dent. 1992; 16(2):119-20.
23.Guttal KS, Naikmasur VG, Bhargava P, Bathi RJ. Fequency of developmental dental anomalies in the Indian population. Eur J Dent. 2010;4(3):263-9.24.

24. Takaoka LA, Goulart AL, Kopelman BI, Weiler RM. Enamel defects in the complete primary dentition of children born at term and preterm. Pediatr Dent. 2011;33(2):171-6.

25.Lygidakis NA. Treatment modalities in children with teeth affected by molar-incisor enamel hypomineralisation (MIH): A systematic review. Eur Arch Paediatr Dent. 2010;11(2):65-74. 26.Mathu-Muju K, Wright JT. Diagnosis and treatment of molar incisor hypomineralisation. Compend Contin Educ Dent. 2006; 27(11): 604-10.
27.Abanto JA, Rezende KMPC, Marocho SMS, Alves FB, Celiberti P, Ciamponi Al. Dental fluorosis: Exposure, prevention and management. Med Oral Patol Oral Cir Bucal. 2009;14(2):103-7.

28.Akpata ES. Occurrence and management of dental fluorosis. Int Dent J. 2001;51(5):325-33.

29.Patel RRA, Hovijitra S, KaFrawy AH, Bixler D. X-linked (recessive) hypomaturation amelogenesis imperfecta: a prosthodontic, genetic, and histopathologic report. J Prosthet Dent. 1991; 66(3): 398-402.

30.Rada RE, Hasiakos PS. Current treatment modalities in the conservative restoration of amelogenesis imperfect: a case report. Quintess Int. 1990; 21(12):937-42 